\title{
EXISTENCE OF SOLUTIONS FOR HIGH ORDER ORDINARY DIFFERENTIAL EQUATIONS WITH SOME PERIODIC-TYPE BOUNDARY CONDITION
}

\author{
S. C. JHUANG, W. C. LIAN, S. P. WANG AND F. H. WONG
}

Abstract. We consider the following high order periodic-type boundary value problem

$(P B V P)\left\{\begin{array}{l}(E) u^{(n)}(t)=f\left(t, u(t), u^{(1)}(t), \cdots, u^{(n-2)}(t), u^{(n-1)}(t)\right) \text { for } t \in(0, T) \\ (P B C)\left\{\begin{array}{l}u^{(i)}(0)=0,0 \leq i \leq n-3, \\ u^{(n-2)}(0)=u^{(n-2)}(T), \\ u^{(n-1)}(0)=u^{(n-1)}(T),\end{array}\right.\end{array}\right.$

where $f \in C\left([0, T] \times \mathbb{R}^{n}, \mathbb{R}\right), n \geq 2$ and satisfies the so-called Nagumo's condition. In this article, we will use a general upper and lower solution method to establish an existence theorem for solutions of $(P B V P)$.

\section{Introduction}

In this paper, we construct an upper and lower solution theory for the existence of solutions of the high order periodic-type boundary value problem as follows:

$(P B V P)\left\{\begin{array}{l}(E) u^{(n)}(t)=f\left(t, u(t), u^{(1)}(t), \cdots, u^{(n-2)}(t), u^{(n-1)}(t)\right) \text { for } t \in(0, T) \\ (P B C)\left\{\begin{array}{l}u^{(i)}(0)=0,0 \leq i \leq n-3, \\ u^{(n-2)}(0)=u^{(n-2)}(T), \\ u^{(n-1)}(0)=u^{(n-1)}(T),\end{array}\right.\end{array}\right.$

where $f \in C\left([0, T] \times \mathbb{R}^{n}, \mathbb{R}\right), n \geq 2$.

Mawhin in [11] first gave the upper and lower solution method for the following Duffing equation

$$
u^{\prime \prime}+k u^{\prime}+f(t, u)=0 \text { on }(0, T)
$$

with boundary condition

$$
u(0)=u(T), \quad u^{\prime}(0)=u^{\prime}(T)
$$

Corresponding author: S. P. Wang.

Received September 16, 2009; revised May 3, 2010.

2000 Mathematics Subject Classification. 34B15.

Key words and phrases. Periodic-type boundary condition, upper and lower solutions, Nagumo's condition, Leray-Schauder degree theory. 
under the continuity of $f$. Nkashama generalized this method to the Carathéodory case in [12] for the first order differential equation. In [8], Habets et al. obtained some similar results to the Carathéodory case for Liénard equation, which is more general than the Duffing equation. But their results are only applicable to the case $k>0$. In [13], Nieto et al. extended these results in a way. Moreover, Wang in [14] extended these results under a Carathéodory case for $k \in \mathbb{R} \backslash\{0\}$, the upper and lower solutions which he used may no longer be periodic. Recently, more and more authors pay attention to second order periodic boundary value problems, such as $[9,15]$. In this article, we want to generalize the upper and lower solution theory for the existence of second order periodic BVPs, mentioned in [6, Theorem 2.1].

In 1934, J. Leray and J. Schauder introduced some "Nonlinear Alternative" theorems for compact maps. These theorems have enhanced greatly the theory of ordinary differential equations and are still used widely today. In this article, we attempt to generalize the above-mentioned results with respect to the existence of solutions of second order periodic boundary value problems to high order periodic-type boundary value problems $(P B V P)$. In fact, there are many investigations extended the related topics about second order differential equations to high order differential equations. For these investigations, we refer to Agarwal et al. $[1,2,3,4]$ and the references therein.

This paper is organized as follows: In this section, we give an brief introduction for our motivation. The section 2 offers some definitions and preliminaries. Our main result is stated in our section 3, which is constructed by upper and lower solution method associated with the related degree theory. Finally, we afford an example for applications.

\section{Definitions and preliminaries}

First, we introduce some extension on the well-known Nagumo's condition[5] of our nonlinear source term.

Definition A. Let $f \in C\left([0, T] \times \mathbb{R}^{n}, \mathbb{R}\right)$ and $v, w \in C^{n}([0, T], \mathbb{R})$ with $v^{(n-2)}(t) \leq$ $w^{(n-2)}(t)$ on $[0, T]$. Then we say that $f$ satisfies a Nagumo's condition on $[0, T]$ with respect to $(v, w, h, N)$, if the following hypotheses hold:

(i) there exists a function $h \in C([0, \infty),(0, \infty))$ such that

$$
\left|f\left(t, p_{1}, \cdots, p_{n-1}, p_{n}\right)\right| \leq h\left(\left|p_{n}\right|\right), \text { for } t \in[0, T], v^{(i-1)}(t) \leq p_{i} \leq w^{(i-1)}(t),
$$

where $i=1,2, \ldots, n-1$;

(ii) there is an $N>0$ such that $\left|v^{(n-1)}(t)\right|,\left|w^{(n-1)}(t)\right| \leq N$ on $[0, T]$ and

$$
\int_{0}^{N} \frac{s}{h(s)} d s>\max _{t \in[0, T]} w^{(n-2)}(t)-\min _{t \in[0, T]} v^{(n-2)}(t)
$$

Next, we define the corresponding lower and upper solution. 
Definition B. We call $v$ and $w \in C^{n}([0, T], \mathbb{R})$ are lower solution and upper solution of $(P B V P)$ in the following sense, respectively.

$$
\begin{aligned}
& \left(\left(1^{0}\right) v^{(n)}(t) \geq f\left(t, v(t), v^{(1)}(t), \cdots, v^{(n-2)}(t), v^{(n-1)}(t)\right) \text { for } t \in(0, T),\right. \\
& \left(2^{0}\right) w^{(n)}(t) \leq f\left(t, w(t), w^{(1)}(t), \cdots, w^{(n-2)}(t), w^{(n-1)}(t)\right) \text { for } t \in(0, T) \text {, } \\
& \left(3^{0}\right)\left\{\begin{array}{l}
v^{(i)}(0) \leq 0, i=0, \ldots, n-3, \\
v^{(n-2)}(0)=v^{(n-2)}(T),
\end{array}\right. \\
& \left\{3^{0}\right)\left\{\begin{array}{l}
v^{(n-2)}(0)=v^{(n-2)}(T), \\
v^{(n-1)}(0) \geq v^{(n-1)}(T),
\end{array}\right. \\
& \left(4^{0}\right)\left\{\begin{array}{l}
w^{(i)}(0) \geq 0, i=0, \ldots, n-3, \\
w^{(n-2)}(0)=w^{(n-2)}(T), \\
w^{(n-1)}(0) \leq w^{(n-1)}(T) .
\end{array}\right.
\end{aligned}
$$

The following tool is essential as looking for fixed points of maps by means of a "homotopic invariance" type of approach.

Theorem C.([10]). Let $B$ be a Banach space, and $\Omega$ be a bounded open neighborhood of $p \in B$. Let $T: \bar{\Omega} \rightarrow B$ be a continuous and compact map. Define a map $H: \bar{\Omega} \times[0,1] \rightarrow B$ by

$$
H(u, \lambda)=u-\lambda T u, \text { for } u \in \bar{\Omega}, \lambda \in[0,1] .
$$

Suppose that $H(u, \lambda) \neq p$ for all $\lambda \in[0,1], u \in \partial \Omega$. Then the equation:

$$
H(u, 1)=p \text {, that is, } T u=u-p
$$

has a solution $u \in \Omega$.

\section{Main results}

Throughout this paper, we assume: $\left(H_{1}\right) v$ and $w$ are the given lower and upper solutions of $(P B V P)$ with

$$
v^{(n-2)}(t) \leq w^{(n-2)}(t) \text { for } t \in[0, T],
$$

$\left(H_{2}\right) f$ satisfies a Nagumo's condition with respect to $(v, w, h, N)$, and

$\left(H_{3}\right)$ for fixed $\left(t, p_{n-1}\right) \in[0, T] \times\left[v^{(n-2)}(t), w^{(n-2)}(t)\right], f\left(t, p_{1}, \cdots, p_{n-2}, p_{n-1}, p_{n}\right)$ is decreasing with respect to $\left(p_{1}, \cdots, p_{n-2}, p_{n}\right) \in[v(t), w(t)] \times \cdots \times\left[v^{(n-3)}(t), w^{(n-3)}(t)\right] \times \mathbb{R}$.

From $\left(H_{2}\right)$, we define the modification $f^{*}\left(t, p_{1}, \cdots, p_{n-1}, p_{n}\right)$ of $f\left(t, p_{1}, \cdots, p_{n-1}, p_{n}\right)$ associated with the triple $(v(t), w(t), N)$ as follows:

$$
f^{*}\left(t, p_{1}, \cdots, p_{n-1}, p_{n}\right):=f\left(t, \eta_{1}, \eta_{2}, \cdots, \eta_{n-1}, \eta_{n}\right)+p_{n-1}-\eta_{n-1},
$$

where, for $i=1,2, \ldots, n-1, t \in[0, T]$,

$$
\eta_{i}= \begin{cases}w^{(i-1)}(t), & \text { if } p_{i}>w^{(i-1)}(t), \\ p_{i}, & \text { if } v^{(i-1)}(t) \leq p_{i} \leq w^{(i-1)}(t), \\ v^{(i-1)}(t), & \text { if } p_{i}<v^{(i-1)}(t),\end{cases}
$$


and

$$
\eta_{n}=\left\{\begin{array}{l}
N, \quad \text { if } p_{n}>N, \\
p_{n}, \quad \text { if }-N \leq p_{n} \leq N, \\
-N, \text { if } p_{n}<-N .
\end{array}\right.
$$

Note that $f^{*}$ is bounded on $[0, T] \times \mathbb{R}$. Immediately, we can define the modified periodic boundary value problem of $(P B V P)$

$\left(P^{*}\right)\left\{\begin{array}{l}\left(E^{*}\right) u^{(n)}(t)=f^{*}\left(t, u(t), u^{(1)}(t), \cdots, u^{(n-2)}(t), u^{(n-1)}(t)\right) \text { for } t \in(0, T) \\ (P B C)\left\{\begin{array}{l}u^{(i)}(0)=0,0 \leq i \leq n-3, \\ u^{(n-2)}(0)=u^{(n-2)}(T), \\ u^{(n-1)}(0)=u^{(n-1)}(T),\end{array}\right.\end{array}\right.$

where $f^{*}$ is the modification of $f$ associated with the triple $(v(t), w(t), N)$ defined as (1).

Lemma 1. Under assumptions $\left(H_{1}\right),\left(H_{2}\right)$ and $\left(H_{3}\right)$, if $u \in C^{n}([0, T], \mathbb{R})$ is a solution of $\left(P^{*}\right)$, then $u$ is also a solution of $(P B V P)$.

Proof. Suppose $u$ is a solution of $\left(P^{*}\right)$, it follows from (1) that we suffices to show that, for all $t \in[0, T], i=0,1, \ldots, n-3, n-2$,

$$
v^{(i)}(t) \leq u^{(i)}(t) \leq w^{(i)}(t) \text { and }\left|u^{(n-1)}(t)\right| \leq N
$$

In order to compete the proof, we separate the proof into the following two steps:

Step 1 We claim that $v^{(n-2)}(t) \leq u^{(n-2)}(t) \leq w^{(n-2)}(t)$ for all $t \in[0, T]$. Suppose to the contrary that there exists a $\bar{t} \in[0, T]$ such that $u^{(n-2)}(\bar{t})>w^{(n-2)}(\bar{t})$. Let $H(t):=u^{(n-2)}(t)-w^{(n-2)}(t)$ on $[0, T]$ and $t_{0} \in[0, T]$ satisfy $H\left(t_{0}\right)=\max _{t \in[0, T]} H(t)>0$. Now, we show that $t_{0} \notin(0, T)$. Assume to the contrary that $t_{0} \in(0, T)$, then $H^{\prime}\left(t_{0}\right)=0$. Hence it follows that

$$
u^{(n-2)}\left(t_{0}\right)>w^{(n-2)}\left(t_{0}\right), u^{(n-1)}\left(t_{0}\right)=w^{(n-1)}\left(t_{0}\right)
$$

and

$$
\left|u^{(n-1)}\left(t_{0}\right)\right|=\left|w^{(n-1)}\left(t_{0}\right)\right| \leq N .
$$

Thus, by Definition B and (1) we have

$$
\begin{aligned}
u^{(n)}\left(t_{0}\right) & =f^{*}\left(t, u\left(t_{0}\right), u^{(1)}\left(t_{0}\right), \cdots, u^{(n-3)}\left(t_{0}\right), u^{(n-2)}\left(t_{0}\right), u^{(n-1)}\left(t_{0}\right)\right) \\
& =f\left(t, \eta_{1}, \eta_{2}, \cdots, \eta_{n-2}, \eta_{n-1}, \eta_{n}\right)+u^{(n-2)}\left(t_{0}\right)-\eta_{n-1} \\
& =f\left(t, \eta_{1}, \eta_{2}, \cdots, \eta_{n-2}, w^{(n-2)}\left(t_{0}\right), w^{(n-1)}\left(t_{0}\right)\right)+u^{(n-2)}\left(t_{0}\right)-w^{(n-2)}\left(t_{0}\right) \\
& >f\left(t, w\left(t_{0}\right), w^{(1)}\left(t_{0}\right), \cdots, w^{(n-3)}\left(t_{0}\right), w^{(n-2)}\left(t_{0}\right), w^{(n-1)}\left(t_{0}\right)\right) \\
& \geq w^{(n)}\left(t_{0}\right)
\end{aligned}
$$

This implies $H^{\prime \prime}\left(t_{0}\right)>0$, which contradicts to the fact that $H\left(t_{0}\right)>0$ is the maximum of $H(t)$ in $(0, T)$. Thus, $t_{0} \notin(0, T)$ and $H\left(t_{0}\right)=H(0)=H(T)=\max _{t \in[0, T]} H(t)>0$. Hence, we can see that

$$
0 \geq H^{\prime}(0)=u^{(n-1)}(0)-w^{(n-1)}(0) \geq u^{(n-1)}(T)-w^{(n-1)}(T)=H^{\prime}(T) \geq 0 .
$$


This implies $H^{\prime}(0)=H^{\prime}(T)=0$, that is, $u^{(n-1)}(0)=w^{(n-1)}(0)=u^{(n-1)}(T)=$ $w^{(n-1)}(T)$. Moreover, for $t>0$ near 0 , one have

$$
u^{(n-2)}(s)-w^{(n-2)}(s)>0 \text {, and } u^{(n-1)}(s)-w^{(n-1)}(s) \leq 0 \text {, for all } s \in(0, t) .
$$

Thus, again by Definition B and $\left(H_{3}\right)$,

$$
\begin{aligned}
u^{(n-1)} & \left(\frac{t}{2}\right)-w^{(n-1)}\left(\frac{t}{2}\right)=\int_{0}^{\frac{t}{2}}\left(u^{(n)}(s)-w^{(n)}(s)\right) d s \\
& =\int_{0}^{\frac{t}{2}} f^{*}\left(s, u(s), \cdots, u^{(n-1)}(s)\right)-w^{(n)}(s) d s \\
& \geq \int_{0}^{\frac{t}{2}} f\left(s, w(s), \cdots, w^{(n-1)}(s)\right)+u^{(n-2)}(s)-w^{(n-2)}(s)-w^{(n)}(s) d s \\
& >0
\end{aligned}
$$

a contradiction. So far we already show that $u^{(n-2)}(t) \leq w^{(n-2)}(t)$ on $[0, T]$. Similarly, we can prove that $v^{(n-2)}(t) \leq u^{(n-2)}(t)$ on $[0, T]$. Finally, since

$$
v^{(i)}(0) \leq u^{(i)}(0) \leq w^{(i)}(0), \text { for } i=0,1, \ldots, n-3,
$$

we conclude that

$$
v^{(i)}(t) \leq u^{(i)}(t) \leq w^{(i)}(t) \text { on }[0, T] \text {, for } i=0,1, \ldots, n-3, n-2 .
$$

Step 2 Note that by the mean value theorem, there exists a point $\tilde{t} \in(0, T)$ such that

$$
\left|u^{(n-1)}(\tilde{t})\right|=\left|\frac{u^{(n-2)}(T)-u^{(n-2)}(0)}{T-0}\right|=0 .
$$

We claim that $\left|u^{(n-1)}(t)\right| \leq N$ on $[0, T]$. Suppose to the contrary that $\left|u^{(n-1)}(t)\right|>N$ for some $t \in[0, T]$. Then there exists an interval $\left[t_{1}, t_{2}\right] \subset[0, T]$ such that one of the following cases holds:

Case $\left(1^{0}\right) u^{(n-1)}\left(t_{1}\right)=0, u^{(n-1)}\left(t_{2}\right)=N\left(\right.$ or $\left.u^{(n-1)}\left(t_{1}\right)=N, u^{(n-1)}\left(t_{2}\right)=0\right)$ and $0<u^{(n-1)}(t)<N$ in $\left(t_{1}, t_{2}\right)$;

$\operatorname{Case}\left(2^{0}\right) u^{(n-1)}\left(t_{1}\right)=0, u^{(n-1)}\left(t_{2}\right)=-N\left(\right.$ or $\left.u^{(n-1)}\left(t_{1}\right)=-N, u^{(n-1)}\left(t_{2}\right)=0\right)$ and $-N<u^{(n-1)}(t)<0$ on $\left(t_{1}, t_{2}\right)$.

Let us consider the Case $\left(1^{0}\right)$. Since we have proved that $v^{(i)}(t) \leq u^{(i)}(t) \leq w^{(i)}(t)$ on $[0, T]$ for $i=0,1, \ldots, n-2$, it follows from Definition $\mathbf{A}$ and (1) that

$$
\begin{aligned}
\left|u^{(n)}(t)\right| u^{(n-1)}(t) & =\left|f\left(t, u(t), u^{(1)}(t), \cdots, u^{(n-2)}(t), u^{(n-1)}(t)\right)\right| u^{(n-1)}(t) \\
& \leq h\left(\left|u^{(n-1)}(t)\right|\right) u^{(n-1)}(t)
\end{aligned}
$$


on $\left[t_{1}, t_{2}\right]$. Therefore, we see that

$$
\begin{aligned}
u^{(n-2)}\left(t_{2}\right)-u^{(n-2)}\left(t_{1}\right) & =\int_{t_{1}}^{t_{2}} u^{(n-1)}(s) d s \\
& \geq \int_{t_{1}}^{t_{2}} \frac{\left|u^{(n)}(s)\right| u^{(n-1)}(s)}{h\left(\left|u^{(n-1)}(s)\right|\right)} d s \\
& \geq\left|\int_{t_{1}}^{t_{2}} \frac{u^{(n)}(s) u^{(n-1)}(s)}{h\left(\left|u^{(n-1)}(s)\right|\right)} d s\right| \\
& =\int_{0}^{N} \frac{\theta}{h(\theta)} d s \\
& >\max _{t \in[0, T]} w^{(n-2)}(t)-\min _{t \in[0, T]} v^{(n-2)}(t) \\
& \geq u^{(n-2)}\left(t_{2}\right)-u^{(n-2)}\left(t_{1}\right) .
\end{aligned}
$$

This leads a contradiction. One can deal with Case $\left(2^{0}\right)$ in a similar way and conclude that $\left|u^{(n-1)}(t)\right| \leq N$ on $[0, T]$. Hence, we complete the proof.

Theorem 2(Main Result). Under assumptions $\left(H_{1}\right),\left(H_{2}\right)$ and $\left(H_{3}\right),(P B V P)$ has at least one solution $u \in C^{n}([0, T], \mathbb{R})$ such that, for $i=0,1, \ldots, n-2$,

$$
v^{(i)}(t) \leq u^{(i)}(t) \leq w^{(i)}(t) \text { and }\left|u^{(n-1)}(t)\right| \leq N \text { on }[0, T] .
$$

Proof. It follows from Lemma 1 that we only have to show that the modified problem $\left(P^{*}\right)$ has at least one solution by applying Theorem C. First, for $\lambda \in[0,1]$, we consider

$\left(P_{\lambda}\right)\left\{\begin{array}{l}u^{(n)}(t)-u^{(n-2)}(t)=\lambda\left[f^{*}\left(t, u(t), u^{(1)}(t), \cdots, u^{(n-2)}(t), u^{(n-1)}(t)\right)-u^{(n-2)}(t)\right], \\ (P B C) .\end{array}\right.$

Define two operator $L: B_{0} \rightarrow C([0, T], \mathbb{R})$ by

$$
(L u)(t):=u^{(n)}(t)-u^{(n-2)}(t), \quad t \in[0, T],
$$

where $B_{0}:=\left\{u \in C^{n}([0, T], \mathbb{R}) \mid u\right.$ satisfies $\left.(P B C)\right\}$ and $G: C^{n-1}([0, T], \mathbb{R}) \rightarrow C([0, T], \mathbb{R})$ by

$$
(G u)(t)=f^{*}\left(t, u(t), u^{(1)}(t), \cdots, u^{(n-2)}(t), u^{(n-1)}(t)\right)-u^{(n-2)}(t), \quad t \in[0, T] .
$$

Claim: $L^{-1}: C([0, T], \mathbb{R}) \rightarrow B_{0}$ exists and is continuous.

Now we show that $\operatorname{ker}(L)=\{0\}$. It is clear that the equation $u^{(n)}(t)-u^{(n-2)}(t)=0$ has a general solution

$$
u(t)=a_{0}+a_{1} t+\cdots+a_{n-3} t^{n-3}+c_{1} e^{t}+c_{2} e^{-t},
$$


where $a_{0}, a_{1}, \cdots, a_{n-3}, c_{1}, c_{2}$ are constants. Since $u \in B_{0}$, by a simple calculation, we obtain that $a_{0}=a_{1}=\cdots=a_{n-3}=c_{1}=c_{2}=0$ which implies $u(t) \equiv 0$. Thus, $\operatorname{ker}(L)=\{0\}$, that is, $L$ is injective. One can also verify that $L$ is surjective via the method of variation of parameters. Hence, $L^{-1}$ exists. Finally, according to the inverse mapping theorem we conclude that $L^{-1}: C([0, T], \mathbb{R}) \rightarrow B_{0}$ is also continuous and complete this claim.

Let $\Omega=\left\{u \in C^{n-1}([0, T], \mathbb{R}) \mid\|u\|_{C^{n-1}}<\frac{M}{r}\right\}$, where $M>0$ is the bound of $f^{*}$ on $[0, T] \times \mathbb{R}^{n}$, and $r=\frac{1}{\left\|L^{-1}\right\|}<\infty$ since $L^{-1}$ exists and is continuous. $\Omega$ is a bounded open neighborhood of 0 in the Banach space $C^{n-1}([0, T], \mathbb{R})$. Define $T=L^{-1} G$. We can show that $T$ is a compact and continuous map from $\bar{\Omega}$ into $C^{n-1}([0, T], \mathbb{R})$. Let $H(u, \lambda)=u-\lambda T u$ for $u \in \bar{\Omega}, \lambda \in[0,1]$, then $H: \bar{\Omega} \times[0,1] \rightarrow C^{n-1}([0, T], \mathbb{R})$ is of the form as shown in Theorem C. Since $0 \in \Omega$, it is clear that $H(u, \lambda) \neq 0$ when $u \in \partial \Omega$ and $\lambda=0$. For $\lambda=1$, if $H(u, \lambda)=0$ for some $u \in \partial \Omega$, then we already have a solution to $\left(P^{*}\right)$. Hence, we assume here that $H(u, 1) \neq 0$ for $u \in \partial \Omega$. It remains to show that $H(u, \lambda) \neq 0$ for $u \in \partial \Omega, 0<\lambda<1$, before applying Theorem C. Suppose not, then there is a $\tilde{\lambda} \in(0,1), \tilde{u} \in \partial \Omega$ satisfies $\tilde{u}=\tilde{\lambda} T \tilde{u}$, that is,

$$
L \tilde{u}(t)=G \tilde{u}(t) \text { on }[0, T] .
$$

Moreover, since $K:=L^{-1}$ exists and is continuous, we have

$$
M>\left\|\tilde{u}^{(n)}-\tilde{u}^{(n-2)}\right\|_{C^{0}}=\|L \tilde{u}\|_{C^{0}} \geq\left\|\frac{K}{\|K\|} L \tilde{u}\right\|_{C^{n}}=r\|\tilde{u}\|_{C^{n}},
$$

this leads a contradiction. Hence, by Theorem $\mathbf{C}$, there is a $u \in \Omega$ satisfying $T u=u$. In fact, $u \in C^{n}([0, T], \mathbb{R})$ is a solution of $\left(P^{*}\right)$ and we completely prove our main result.

\section{An Example}

Example 3. Consider

$$
\left\{\begin{array}{l}
\epsilon^{3} u^{\prime \prime \prime}(t)=\left(u^{\prime \prime}(t)\right)^{\frac{3}{2}}+\left(u^{\prime}(t)\right)^{3}-\cos ^{\frac{3}{2}} t-\sin ^{3} t \text { on }(0,2 \pi), \\
u(0)=0, u^{\prime}(0)=u^{\prime}(2 \pi), u^{\prime \prime}(0)=u^{\prime \prime}(2 \pi),
\end{array}\right.
$$

where $\epsilon>0$ is a small parameter. It is easy to see that

$$
v(t)=-\cos t-2 \epsilon t-1 \text { and } w(t)=-\cos t+2 \epsilon t+1
$$

are lower and upper solutions. Moreover, $f\left(t, p_{1}, p_{2}, p_{3}\right):=p_{3}^{\frac{3}{2}}+p_{2}^{3}-\cos ^{\frac{3}{2}} t-\sin ^{3} t$ satisfies $\left(H_{1}\right),\left(H_{2}\right)$ and $\left(H_{3}\right)$. By Theorem 2, this problem has a solution

$$
u(t)=-\cos t+O(\epsilon) .
$$

Moreover,

$$
u^{\prime}(t)=\sin t+O(\epsilon) \text { and } u^{\prime \prime}(t)=\cos t
$$




\section{References}

[1] R. P. Agarwal and F. H. Wong, Existence of positive solutions for higher order boundary value problems, Nonlinear Studies 5 (1998), 15-24.

[2] R. P. Agarwal and F. H. Wong, Existence of positive solutions for non-positive higher order BVP's, Computational and Applied Mathematics 88 (1998), 3-14.

[3] R. P. Agarwal and F. H. Wong, An application of topological transervality with respect to non-positive higher order BVP's Applied Mathematics and Computation 99 (1999), $167-$ 178.

[4] R. P. Agarwal, F. H. Wong and W. C. Lian, Existence of positive solutions of nonlinear second order differential systems, Appliciable Analysis 63 (1996), 375-387.

[5] S. R. Bernfeld and V. Lakshmikantham, An introduction to nonlinear boundary value problems, Academic Press Inc., New York and London, 1974.

[6] C.De Coster and P.Habets Upper and lower solutions in the theory of ode boundary value problems: classical and resent results, in "Nonlinear analysis and boundary value problems for ordinary differentail equations" (Edited by F.Zanolin) C.I.S.M. Courses and Lectures 371, Springer, New York (1993), 1-79.

[7] P. Habets and G. Metzen, Existence of periodic solutions of Duffing equations, J. Differential Equations 78 (1989), 1-32.

[8] P. Habets and L. Sanchez, Periodic solutions of some Liénard equations with singularities, Proc. Amer. math. Soc. 109 (1990), 1035-1044.

[9] D. Jiang M. Fan and A. Wan, A monotone method for constructing extremal solutions to second-order periodic boundary value problems, J. Computat. Applied Math. 136 (2001), 189-197.

[10] J. Leray and J. Schauder, Topologie et equations fonctionelles, Ann. Sci. l'Ecole Norm. Sup. 51 (1934), 45-78.

[11] J. Mawhin, Points fixes, points critiques et probléme aux limits, Semin. Math. Sup., 92, Press Univ. de Montreál, 1985.

[12] M. N. Nkashama, A generalized upper and lower solutions method and multiplicity results for nonlinear first-order ordinary differential equations, J. Math. Anal. Applic. 140 (1989), 381-395.

[13] J. J. Nieto, et al, A generalization of the monotone iterative techniqne for nonlinear second order periodic boundary value problem, J. Math. Anal. Applic. 151 (1990), 181-189.

[14] C. Wang, Generalized upper and lower solution method for the forced Duffing equation, Proc. Amer. math. Soc. 125 (1997), 397-406.

[15] F. H. Wong, W. C. Lian and S. C. Chen, Existence of solutions for second order periodic boundary value problems, International J. Pure Applied Math. 16 (2004), 393-400.

Department of Mathematics, National Taipei University of Education, 134, Ho-Ping E. Rd, Sec2, Taipei 10659, Taiwan.

E-mail:

National Kaohsiung Marine University, No. 142, Hai Chuan Road, Nan-Tzu Dist, Kaohsiung, Taiwan.

E-mail: wclian@mail.nkmu.edu.tw 
Holistic Education Center, Cardinal Tien College of Healthcare and Management, 171, Jhongsing Rd., Sansing, Yilan 26646, Taiwan.

E-mail: spwang@ctcn.edu.tw

Department of Mathematics, National Taipei University of Education, 134, Ho-Ping E. Rd, Sec2, Taipei 10659, Taiwan.

E-mail: wong@tea.ntue.edu.tw 